\title{
Efficacy and safety of subthreshold micropulse laser compared with threshold conventional laser in central serous chorioretinopathy
}

\author{
Zuhua Sun ${ }^{1}$ Ying Huang ${ }^{1} \cdot$ Chaochao Nie ${ }^{1} \cdot$ Zhijie Wang $^{1} \cdot$ Junqing Pei ${ }^{1} \cdot$ Bing Lin $^{1} \cdot$ Rong Zhou $^{1} \cdot$ Junyan Zhang ${ }^{2}$. \\ Victor Chong $\mathbb{1 0}^{3,4} \cdot$ Xiaoling Liu ${ }^{1}$
}

Received: 28 March 2019 / Revised: 29 September 2019 / Accepted: 31 October 2019 / Published online: 29 November 2019

(c) The Author(s) 2019. This article is published with open access

\begin{abstract}
Purpose To compare the efficacy and safety of subthreshold micropulse laser (SML) with threshold conventional laser (TCL) in central serous chorioretinopathy (CSC).

Methods Prospective, randomized, double-masked, non-inferiority, 12-week clinical trial. Patients were randomly assigned 1:1 to SML group or TCL group. Patients in the SML group were treated with $577 \mathrm{~nm}$ micropulse laser. The spot size was $160 \mu \mathrm{m}$, the duty cycle was $5 \%$ and exposure time was $0.2 \mathrm{~s}$. The power was $50 \%$ threshold tested. Patients in the TCL group were treated with $577 \mathrm{~nm}$ continuous laser. The power was $100 \%$ threshold tested. The primary outcome was the mean change in best-corrected visual acuity (BCVA) at week 12, with a non-inferiority limit of five letters on the Early Treatment Diabetic Retinopathy Study (ETDRS) visual acuity charts.

Results Eighty-eight patients were enroled. Seventy-seven patients were male. Forty-four patients were in SML group and 44 in TCL group. At week 12, SML was equivalent to TCL with a gain of $6.23 \pm 8.59$ and $6.61 \pm 6.35$ letters, respectively, (SML-TCL difference: -0.38 letters; $95 \%$ confidence interval $(\mathrm{CI}):-3.58-2.81 ; P_{\text {non-inferiority }}=0.0026$ ). There was no statistically significant difference between the two groups $(t=0.240, P=0.811)$. At week 12 , the proportion of patients whose SRF had been totally absorbed was 63.63 and $81.82 \%$ respectively for SML and TCL groups. There was no statistically significant difference between the two groups $\left(\chi^{2}=3.67, P=0.056\right)$.

Conclusions Both SML and TCL can improve visual acuity in CSC. SML was non-inferior to TCL in the improvement of BCVA.
\end{abstract}

\section{Introduction}

Central serous chorioretinopathy (CSC) is a relatively common early-onset disease characterized by serous detachment of the neural retina with dysfunction of the choroid and retinal pigment epithelium (RPE). One or more focal leakages of RPE decompensation that have been identified by mid-phase fundus fluorescein angiography

Xiaoling Liu

drliuxiaolin@163.com

1 School of Ophthalmology \& Optometry and Eye Hospital, Wenzhou Medical University, 270 Xueyuan Road,

Wenzhou, Zhejiang, China

2 Bothwin Clinical Study Consultant, Redmond, WA, USA

3 Optegra Eye Hospital, London, UK

4 Royal Free Hospital, London, UK
(FFA) are considered the principal sources of subretinal fluid (SRF) [1,2].

Conventional laser photocoagulation and photodynamic therapy (PDT) are effective methods for treatment of CSC $[3,4]$. Conventional laser photocoagulation is a precise application of laser treatment at a suprathreshold power that creates grade I burns. It is an effective and routine intervention in CSC. Threshold conventional laser (TCL), which may be safer [5], uses less power than the grade I burns. However, both conventional laser and TCL are not appropriate for juxtal or subfoveal leakage because they may cause central or paracentral scotomas, contrast sensitivity loss, accidental foveal damage, retinal distortion and choroidal neovascularization (CNV) as well as atrophic scars on the RPE [6-8]. PDT uses a laser to activate a dye locally, leading to choroidal vascular thrombosis [9]. PDT is an option not only for chronic CSC with diffuse leakage but also for acute CSC with leakage within or close to the fovea [3, 10-12]. While it has not been approved for health 
insurance in China, and it is very expensive. Under these circumstances, PDT has been limited in China.

Unlike conventional laser, a subthreshold micropulse laser (SML) is non-damaging, the para-fovea or fovea can be directly treated, and treatment can be repeated without detectable structural or functional damage [13]. SML involves a series of repetitive laser pulses with an interval. SML have been used in the treatment of a wide range of retinal disease in clinical studies and are commercially available and FDA approved [13], meaning that SML can be considered as an alternative treatment for CSC.

Although SML has been reported in the literature treatment of CSC, the parameters used have been controversial. Several studies using $810 \mathrm{~nm}$ micropulse lasers have demonstrated efficacy in the treatment of CSC [14-20]; however, only a few studies have been conducted using $577 \mathrm{~nm}$ micropulse lasers, and all were done retrospectively [21-24]. The $577 \mathrm{~nm}$ wavelength occurs outside the absorption spectrum of retinal xanthophyll and has major advantages, such as peak absorption of oxyhaemoglobin, minimal xanthophyll absorption in the macula and better penetration, potentially allowing for treatment close to the fovea $[25,26]$. This study is the first prospective, randomized controlled trial comparing the efficacy and safety of $577 \mathrm{~nm}$ SML with $577 \mathrm{~nm}$ TCL in the treatment of CSC.

\section{Subjects and methods}

This was a single-centre, prospective, randomized, double-masked, non-inferiority, 12-week clinical trial comparing the efficacy and safety of SML with TCL in the treatment of CSC. The study was registered with ClinicalTrials.gov (identifier no. NCT02735213). All patients were recruited from the School of Ophthalmology \& Optometry and Eye Hospital, Wenzhou Medical University. The study protocol was approved by the ethics committee of School of Ophthalmology \& Optometry and Eye Hospital, Wenzhou Medical University. The study was carried out in compliance with the Declaration of Helsinki and the International Conference on Harmonization Guidelines for Good Clinical Practice. All patients provided written informed consent to participate in this trial. The data described here were collected between April 2016 and February 2017.

\section{Participants}

Patients $\geq 18$ years with CSC causing SRF involving the centre of the macula within 6 months were eligible for enrolment if central retinal thickness (CRT) measured by spectral-domain optical coherence tomography (SD-OCT)
(Spectralis; Heidelberg Engineering, Heidelberg, Germany) was $\geq 250 \mu \mathrm{m}$, best-corrected visual acuity (BCVA) was at least 64 Early Treatment Diabetic Retinopathy Study (ETDRS) letters (20/50 Snellen equivalent), and the leakage points on FFA were limited in ETDRS ring 2 or 3 (Fig. 1a). Only one eye from each patient was included.

Exclusion criteria were: PDT and/or laser treatments in the study eye within 3 months prior to enrolment, and the presence of other retinal lesions such as $\mathrm{CNV}$, polypoidal choroidal vasculopathy or age-related macular degeneration.

Ninety-two patients provided written informed consent. Eighty-eight patients were enroled and randomized 1:1 into the SML or TCL group according to a predetermined randomization scheme provided by a designated, masked statistician. A secure, computer-generated randomization schedule was maintained in concealed envelopes by a study-group member who did not participate in enrolment. Concealed envelopes were revealed by treatment physicians only after eligibility for enrolment had been confirmed and before treatment were given to the patients.

\section{Treatments}

Patients in the SML group were treated with the Supra Scan $577 \mathrm{~nm}$ micropulse laser mode (Quantel Medical SubLiminal laser). The laser parameter was set to $5 \%$ duty cycle, spot size was $160 \mu \mathrm{m}$, 9-spot matrix without spacing between two spots was used (Fig. 1b). On the micropulse mode, the threshold power was titrated at the arch area. SML was performed on the leakage areas on a mid-phase FA image with the $50 \%$ threshold power $[9,21]$. The total laser burns made were 150-200.

Patients in the TCL group were treated with the Supra Scan $577 \mathrm{~nm}$ continuous laser mode (Quantel Medical, France). The laser parameter was set to the time was $0.05 \mathrm{~s}$, spot size was $100 \mu \mathrm{m}$, and 9-spot matrix with one burn space (Fig. 1c). On the conventional laser mode, the threshold power was titrated near the arch area. TCL was then performed on the leakage areas on a mid-phase FA image with the threshold power tested. The laser burns made were $18-27$.

All the treatments were performed by a single investigator. Prior to treatment, all patients underwent ophthalmic examinations, including BCVA, anterior segment examination, dilated fundus examination, fundus photography (FP) (Canon, CR-1 Mark II, Japan), SD-OCT, FA and indocyanine green angiography (ICGA) (Spectralis, Heidelberg Engineering, Heidelberg, Germany). Patients were re-assessed at 3, 7 and 12 weeks after treatment. BCVA, fundus and OCT examinations were performed. The followup flowchart was outlined in Fig. 2. 
Fig. 1 a FFA of mid-phase showed the leakage located at the ring 2. b Stimulated image of the subthreshold micropulse laser mode. The white square frames represented the treatment area. The red dots represented the laser points. c Stimulated image of the threshold conventional laser mode.
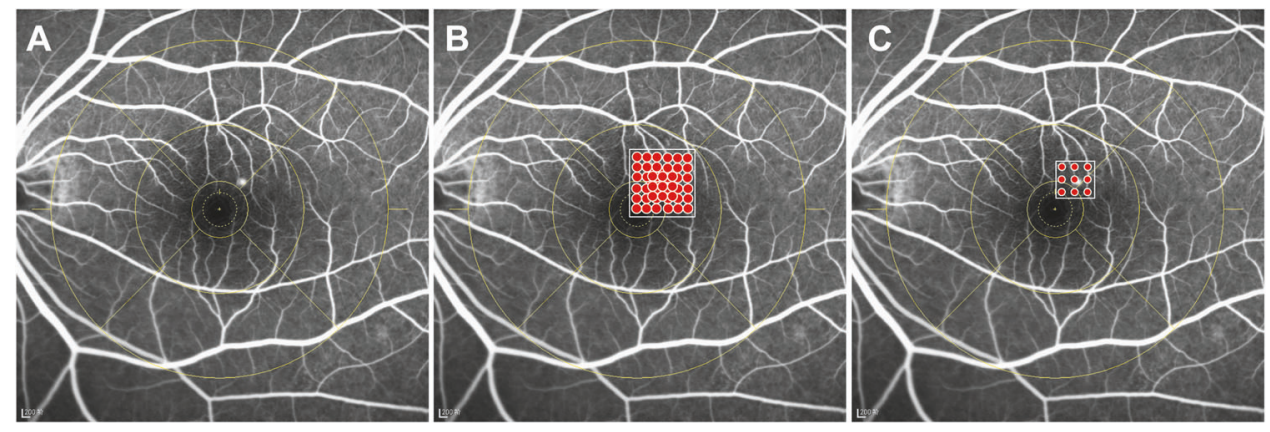

Fig. 2 Study follow-up flow chart. SML Subthreshold micropulse laser, TCL threshold conventional laser.

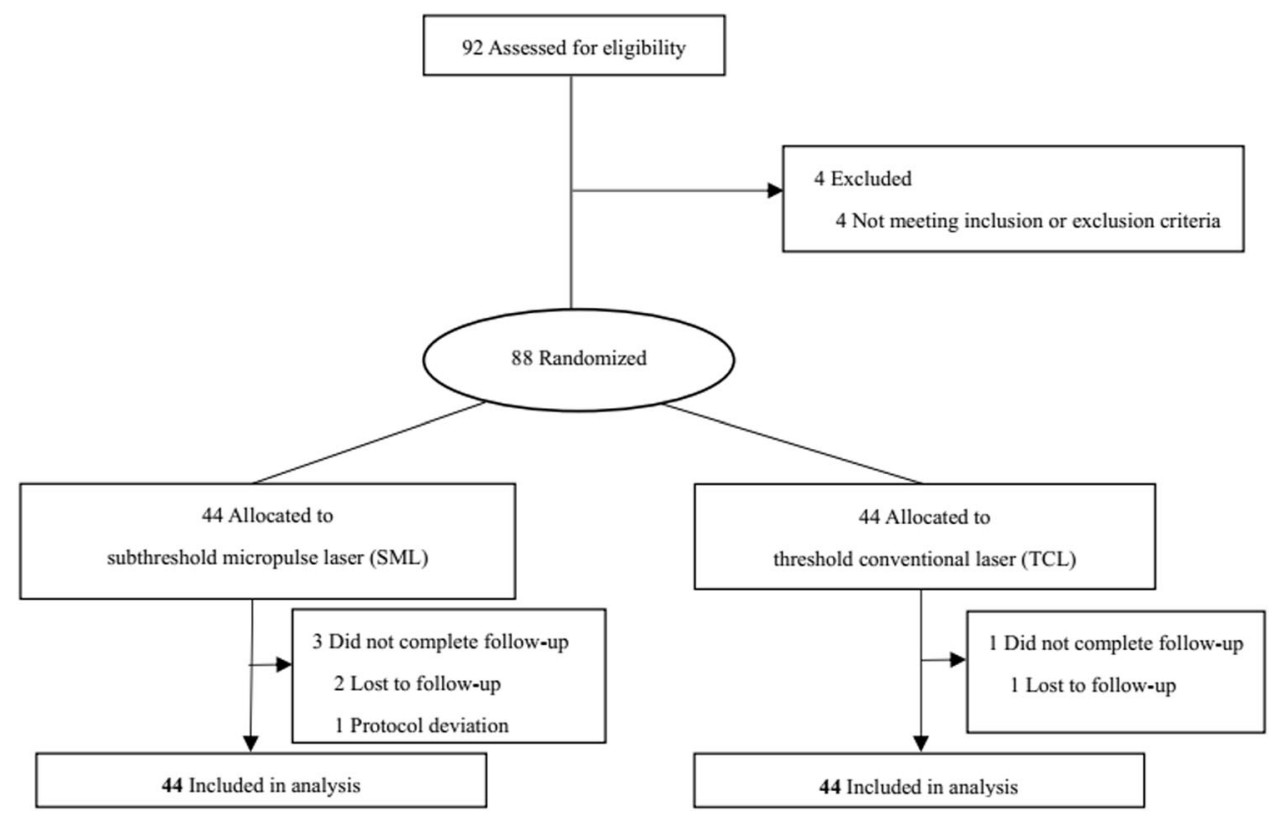

\section{Outcome measurements}

The primary efficacy outcome was change in BCVA from baseline to week 12 . The secondary were the proportion of patients who had totally absorbed SRF at week 12 , changes in BCVA, CRT and central choroidal thickness from baseline to week 3, 7 and 12, respectively. Additional outcomes included the proportion of eyes that gained $\geq 5$ ETDRS letters in BCVA from baseline to week 12, as well as the proportion of eyes that accepted retreatment at week 12. Safety assessments included laser-induced scarring detection on FP at week 12, and any other ocular or systemic event during the 12 weeks of follow-up.

BCVA was assessed following the ETDRS protocol [27] by the certified masked optometrist. SRF, CRT and central choroidal thickness were assessed using SD-OCT by two qualified masked technicians. The OCT examination included 25 sections, each of which comprised nine averaged scans and which were obtained in an area of a $6 \mathrm{~mm} \times$ $6 \mathrm{~mm}$ square centred on the fovea. Central choroid thickness was measured using EDI mode on SD-OCT. The leakage located at ring 2 or 3 on the FA image was assessed following the ETDRS protocol [28]. All of them except the physician who administered the laser treatment were unaware of the patient's treatment assignment.

RPE atrophy was specified as RPE defect, pigmentation was specified as more pigment than usual and migration was specified as pigment displacement by FP.

\section{Statistical analysis}

This study was designed as a non-inferiority trial comparing the two groups. For the primary outcome, non-inferiority limit for the difference between two groups in the mean change in BCVA at week 12 was five letters [29]. Assuming a standard deviation (SD) for changes in BCVA of ten letters, an overall one-sided type I error rate of 0.025 , a rate of loss to follow-up of $20 \%$ and a power of $80 \%$, we determined that a sample of 35 patients per group, adjusted to 44 each. 
A one-tailed statistical test for non-inferiority between the two groups was performed. The primary analysis followed the intention-to-treat principle. Missing data were imputed using the last-observation-carried-forward method. Statistical analysis of the data between baseline and followup in each group was performed. Means \pm SD are reported. $P$ values and confidence intervals $(\mathrm{CI})$ are two sided. $P$ values less than 0.05 were considered statistically significant. Mean values between groups in continuous variables were compared using independent $t$-tests. Changes in BCVA, CRT and central choroidal thickness of both groups from baseline to follow-up were compared using paired $t$-tests and the Wilcoxon rank-sum (Mann-Whitney) test. Chi-square was used to compare categorical data between the two groups.

\section{Results}

Eighty-eight patients were randomized into the SML group $(n=44)$ and TCL group $(n=44)$. Minor differences in baseline clinical characteristics existed between the two groups, as outlined in Table 1. During the study, no patient was using any oral, topical, inhaled or injected corticosteroids. Four patients dropped out of the study without explanation: three in the SML group and one in TCL group. The proportion of patients who completed the study was $95 \%$.

Visual acuity improved from baseline to 12 weeks in both groups (Fig. 3a). At week 12, the changes in BCVA were not statistically significant between the groups $(P=$ $0.811, t$-test). SML was equivalent to TCL with a gain of $6.23 \pm 8.59(P<0.001)$ and $6.61 \pm 6.35(P<0.001)$ letters, respectively, in the full analysis set (SML-TCL difference, -0.38 letters; $95 \% \mathrm{CI},-3.58-2.81 ; P_{\text {non-inferiority }}=0.0026$ ) (Fig. $3 b$ ) and with a gain of $5.55 \pm 8.45$ and $5.45 \pm 5.26$ respectively by intention-to-treat analysis (SML-TCL difference, 0.09 letters; $95 \% \mathrm{CI},-2.89-3.07 ; P_{\text {non-inferiority }}=$ 0.0005). At week 12, the mean BCVA was $82.82 \pm 7.71$ and $83.45 \pm 6.40$ letters in the SML group and in the TCL group, respectively. The proportion of patients who gained $\geq 5$ letters from baseline to week 12 was $68 \%$ in the SML
Table 1 Patient demographics and baseline characteristics.

\begin{tabular}{|c|c|c|c|c|c|}
\hline & SML $(n=44)$ & $\mathrm{TCL}(n=44)$ & $t$ or $\chi^{2}$ or $z$ & P1 & $\mathrm{P} 2$ \\
\hline \multicolumn{6}{|l|}{ Sex } \\
\hline Male, $n(\%)$ & $35(79.55)$ & $42(95.45)$ & & & \\
\hline Female, $n(\%)$ & $9(20.45)$ & $2(4.55)$ & 5.091 & $0.024 *$ & \\
\hline \multicolumn{6}{|l|}{ Age (years) } \\
\hline Mean \pm SD & $44.41 \pm 8.71$ & $44.68 \pm 6.77$ & 0.164 & 0.87 & \\
\hline Median (IQR) & $44(39-51.5)$ & $45(40-48)$ & 0.322 & & 0.748 \\
\hline \multicolumn{6}{|l|}{ Duration (weeks) } \\
\hline Mean \pm SD & $10.50 \pm 8.22$ & $10.20 \pm 8.22$ & -0.169 & 0.867 & \\
\hline Median (IQR) & $7.5(4-18)$ & $6.5(4-18)$ & -0.324 & & 0.746 \\
\hline \multicolumn{6}{|l|}{ BCVA (ETDRS letters) } \\
\hline Mean \pm SD & $76.60 \pm 7.00$ & $77.84 \pm 7.70$ & 0.159 & 0.874 & \\
\hline Median (IQR) & $77(73-80)$ & $77.5(72-82)$ & 0.209 & & 0.835 \\
\hline \multicolumn{6}{|l|}{$\mathrm{CRT}(\mu \mathrm{m})$} \\
\hline Mean \pm SD & $398.50 \pm 92.92$ & $407.49 \pm 133.55$ & 0.367 & 0.715 & \\
\hline Median (IQR) & $381(332-445.75)$ & $374(324.5-458)$ & -0.142 & & 0.887 \\
\hline \multicolumn{6}{|l|}{ Leakage location } \\
\hline Ring $2, n(\%)$ & $35(79.55)$ & $34(77.27)$ & & & \\
\hline Ring 3, $n(\%)$ & $9(20.45)$ & $10(22.73)$ & 0.067 & 0.796 & \\
\hline \multicolumn{6}{|l|}{ Leakage point } \\
\hline Single, $n(\%)$ & $35(79.55)$ & $35(79.55)$ & & & \\
\hline Multiple, $n(\%)$ & $9(20.45)$ & $9(20.45)$ & 0 & 1 & \\
\hline \multicolumn{6}{|l|}{ Primary/recurrent } \\
\hline Primary, $n(\%)$ & $41(93.18)$ & $37(84.09)$ & 1.805 & 0.179 & \\
\hline Recurrent, $n(\%)$ & $3(6.82)$ & $7(15.91)$ & & & \\
\hline Prior laser treatment $n(\%)$ & $1(2.27)$ & $4(9.09)$ & 0.848 & 0.357 & \\
\hline Systemic steroid $n(\%)$ & $0(0)$ & $2(4.55)$ & 0.512 & 0.474 & \\
\hline
\end{tabular}

Full analysis set. P1 represented the $P$ value of the independent $t$-test result or $\chi^{2}$. P2 represented the $P$ value of the Wilcoxon rank-sum (Mann-Whitney) test

SML subthreshold micropulse laser, TCL threshold conventional laser, SD standard deviation, IQR interquartile range, BCVA best-corrected visual acuity, ETDRS Early Treatment Diabetic Retinopathy Study, $C R T$ central retinal thickness

* Significant $P$ value $(<0.05)$ 
A

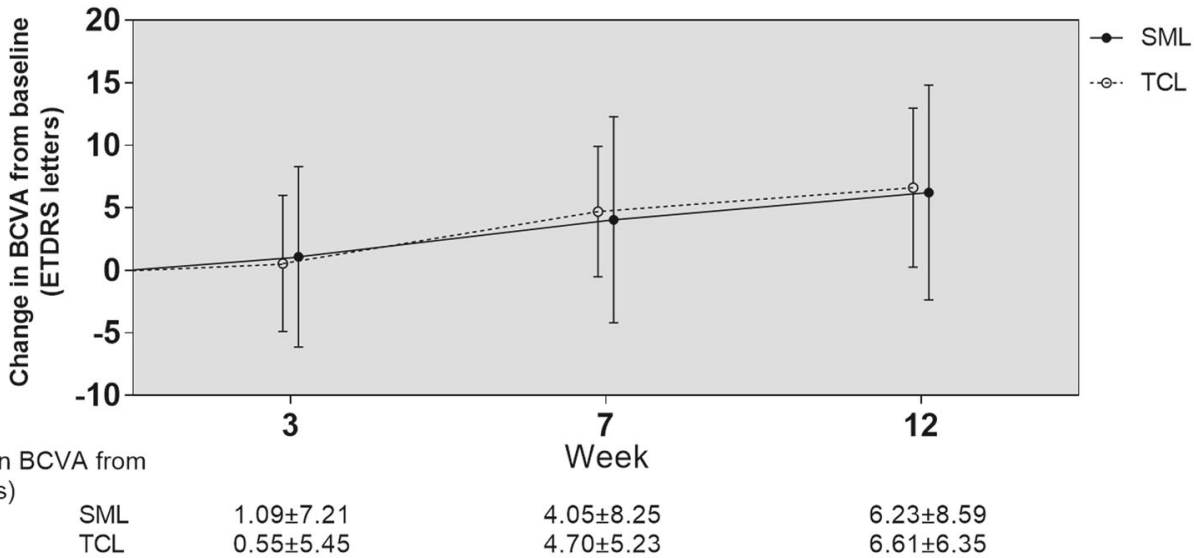

Mean $( \pm S D)$ change in BCVA from Baseline(no. of letters)

TCL $\quad 0.55 \pm 5.45$

$4.70 \pm 5.23$

B

ference in mean change in BCVA at Week 12(no. of letters)

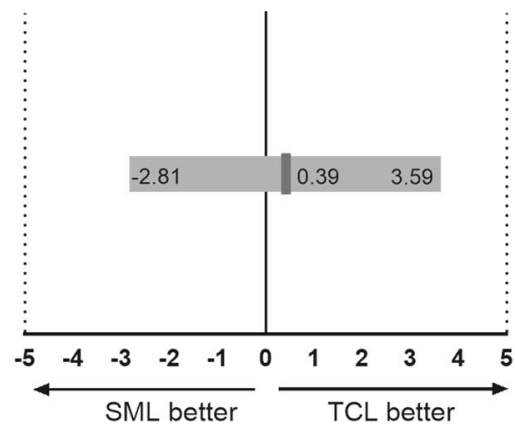

C

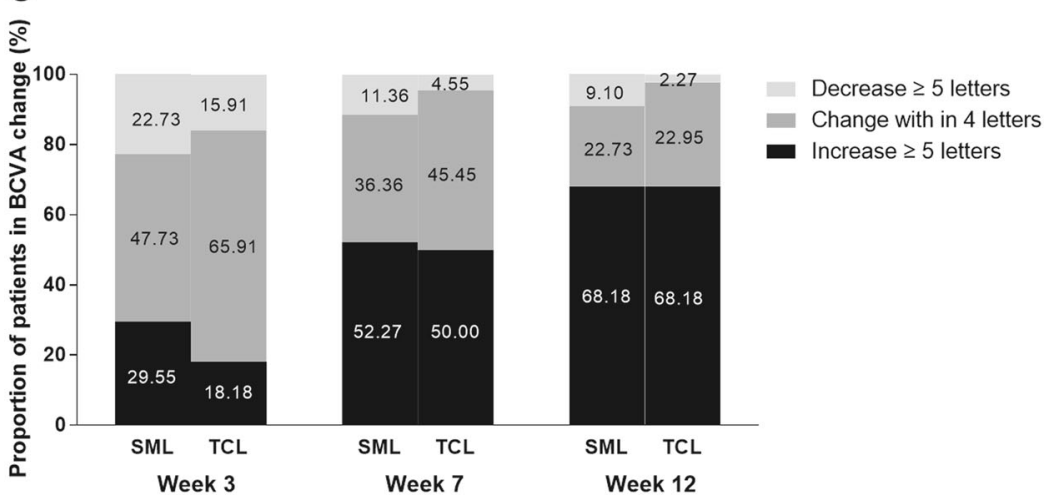

Fig. 3 a Mean change in BCVA. b Differences in BCVA change from baseline to week 12 between the two groups. The red vertical lines indicated the mean difference between the two groups, and the grey bar was the $95.0 \%$ CI. CI within -5 and +5 letters (dashed vertical lines) indicated that the two groups were equivalent. A lower limit of the $95.0 \% \mathrm{CI}$ with a value above -5 showed that SML was noninferior compared with TCL. c Proportions of patients with BCVA change from baseline to week 12. BCVA best-corrected visual acuity, CI confidence interval, SML subthreshold micropulse laser, TCL threshold conventional laser. group, the same as that in the TCL group $(P=1.0$, Chisquare test) (Fig. 3c).

At week 12, all the BCVA scores were over baseline except six patients in the SML group and seven in the TCL group (Fig. 4a). BCVA lost 24 letters from baseline in one patient was because of secondary CNV after the TCL treatment. BCVA lost 15 letters from baseline in one patient in the TCL group because the CRT was only $203 \mu \mathrm{m}$, which was thinner than normal. BCVA lost within 10 letters in the other 11 patients was either because of persistent SRF or because of disorder of the photoreceptor layer.

The proportion of patients whose SRF had been totally absorbed at week 12 was $63.63 \%$ for the SML group, and $81.82 \%$ for the TCL group. There was no statistically significant difference between the two groups at week 12 $(P=0.056)$.

The mean reduction of CRT from baseline to week 3 was $101.39 \pm 97.03 \mu \mathrm{m}$ in the SML group and $140.92 \pm 113.68$ $\mu \mathrm{m}$ in the TCL group. Mean reduction at week 7 was $126.47 \pm 90.05 \mu \mathrm{m}$ in the SML group and 152.66 \pm $131.50 \mu \mathrm{m}$ in the TCL group. The mean reduction at week 12 was $131.66 \pm 79.26 \mu \mathrm{m}$ in the SML group $(P<$ $0.001, t$-test) and $163.00 \pm 136.90 \mu \mathrm{m}$ in the TCL group $(P<0.001)$. There was no statistically significant difference between groups $(P=0.192)$. At week 12 , CRT decreased from baseline except for three patients in the SML group; all members of the TCL group showed a decrease (Fig. 4b).

Central choroidal thickness at baseline was not available for two patients, meaning that data from 86 patients were analysed. The mean reduction of central choroidal thickness from baseline to week 12 was $22.98 \pm 54.94 \mu \mathrm{m}$ in the SML group and $42.94 \pm 46.08 \mu \mathrm{m}$ in the TCL group $(P=0.009$; $P<0.001 ; t$-test, respectively). There was no statistically significant difference between groups in the change of central choroidal thickness at week $12(P=0.071)$.

\section{Safety}

Secondary CNV occurred in one patient in the TCL group. No RPE atrophy, pigmentation, migration or systemic adverse events were observed in the other 87 patients. No laser-induced scarring was detected and only mild RPE 
A

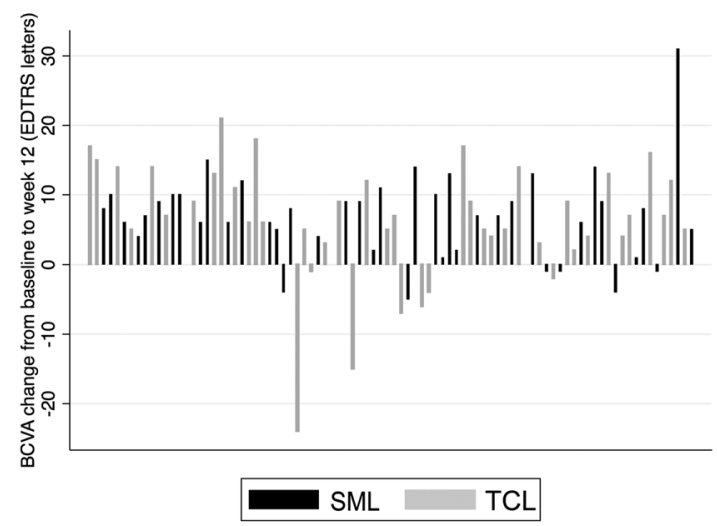

Fig. 4 a Waterfall plots of BCVA changes from baseline to week 12 for individual patients. These plots showed that all the BCVA scores improved except six patients in SML group and seven patients in TCL group. b Waterfall plots of CRT changes from baseline to week 12 for individual patients. These plots showed that all the CRT thicknesses

depigmentation was detected in $12 \%$ patients in the SML group at week 12 by FP images, whereas laser-induced changes were detected in $33 \%$ of patients in the TCL group. The difference between the groups was statistically significant $(P=0.032)$.

\section{Discussion}

Comparing $577 \mathrm{~nm}$ SML and $577 \mathrm{~nm}$ TCL, the effects of SML on visual acuity at 12 weeks were non-inferior to TCL treatment. The proportion of patients who gained at least five letters, the proportion of total SRF absorption and the retreatment rate were practically the same for each group.

Low power (5\% duty cycle, $50 \%$ power of threshold) and high density (150-200 confluent spots in the leakage area) SML for CSC were used in this study. Not only can this effectively accelerate recovery, but it can also improve BCVA. The mechanism of action of SML for CSC was hypothesised as biological activation of the decompensated RPE cells in the leakage area by subtle thermal stress without causing RPE or photoreceptor cell death.

In this study, SML was non-inferior to TCL in terms of BCVA improvement at week 12. Interestingly, the curve of the change in BCVA from baseline differed from the curve of the change in CRT from baseline, which dropped sharply at 3 weeks after SML. The improvement of BCVA was slower than the reduction of CRT. It is likely that the photoreceptor cells need time to recover after the neural retina reattachment and total absorption of SRF have taken place.

SML activated the RPE without damaging RPE or photoreceptors. There was a number of other interventional case series showing complete resolution of SRF
B

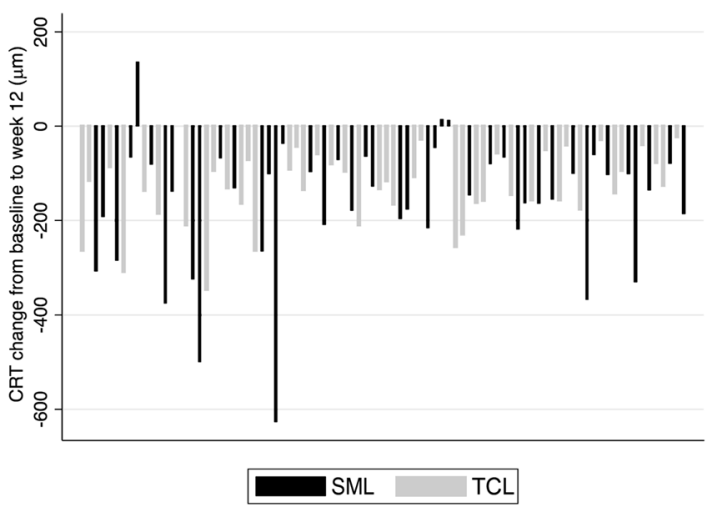

decreased from baseline except three patients in the SML group, but none in TCL group. BCVA best-corrected visual acuity, CRT central retinal thickness, SML subthreshold micropulse laser, TCL threshold conventional laser.

were between 55 and $79 \%$ of patients treated with SML and at least partial resolution in $75-100 \%$ of patients over 2-14 months' follow-up [8, 18, 20, 23, 30, 31]. Scholz et al. reported that only $24 \%$ of patients dried up totally in the retrospective study with $577 \mathrm{~nm}$ SML after the treatment of chronic CSC [21]. SRF had been totally resolved in about $50 \%$ patients at week 3 and $65 \%$ at week 12 after the $577 \mathrm{~nm}$ SML treatment in our study, which was better than Scholz. This more favourable outcome is perhaps due to short disease duration (within 6 months); most patients were also naive in this study. In the TCL group, the proportion of SRF had totally resolved was much higher than in the SML group, although this difference was not statistically significant. If complete SRF absorption after a single treatment is the major aim, TCL $577 \mathrm{~nm}$ is the preferred treatment.

The effect of SML treatment for CSC in the different investigations varies according to different heterogeneous inclusion criteria, laser parameters and laser spot performance area. Some authors adjust laser power upward to the minimum threshold value for a visible burn in a continuous wave mode and then switch the apparatus to micropulse mode $[15,18,23]$. Other researchers have also used the micropulse mode for power titration and applied $50-80 \%$ of the minimum threshold power to cause a barely visible burn [19, 22]. The latter power adjustment method was used in this study. Some authors determine the area to be treated as active leakage on mid-phase FA or hyperfluorescent areas on midphase ICGA images [14, 21, 23, 32]. Malik et al. determine the area to be treated as limited to one disk diameter surrounding fluorescein leakage or the areas of serous elevation as determined by clinical examination and OCT [31]. Kim et al. delivered laser shots over the entire area of CSC, 
including the leaking point on FA and the foveal centre, and also on the normal retina around the borderline area of serous retinal detachment [22]. The area to be treated was determined as active leakage on mid-phase FA or hyperfluorescent areas on mid-phase ICGA images in this study. Further studies are needed to determine a standard power titration protocol and treatment area in SML applications.

SRF had totally resolved in about two thirds of patients, with no laser-induced scarring, pigmentation, CNV or atrophy, at 12 weeks after SML treatment. This indicates that the parameters including laser spots and density of SML for CSC were effective and safe in this study. For the remaining third of patients, we speculate that the intensity of the laser was not enough to fully activate the decompensated RPE. The retreatment rate was $11 \%$ at week 12 in the SML group, which was similar to the TCL group. The result was better than in Scholz's study, which had a retreatment rate of 55\% (39\% twice and $16 \%$ three times) [21]. This could be due to patients who were chronic, including some persistent SRF after PDT in Scholz.

No systemic adverse events were observed in each group. Secondary CNV in one patient and some laser-induced scarring was detected in the TCL group. No scarring was detected in the SML group, suggesting that SML was safer than TCL.

The main limitation of this study is that the follow-up period is relatively short with only 12 weeks. Recurrences are relatively common in CSC, so a longer follow-up period might add much value in the future studies. Another limitation is the absence of an untreated control group or a halfdose PDT group, so it was difficult to determine whether the results were due to the laser treatment or spontaneous resolution because the disease durations in this study were all within 6 months.

In conclusion, the $577 \mathrm{~nm}$ SML was effective and safe in CSC patients, and it was non-inferior to the $577 \mathrm{~nm}$ TCL in the improvement of BCVA. In the cases where leakage is very near to the fovea, SML may be preferred to TCL.

\section{Summary}

\section{What was known before}

- TCL is usually performed in the treatment of CSC

\section{What this study adds}

- SML is non-inferior to TCL in CSC treatment

Acknowledgements The authors would like to thank Jingwei Zheng from the School of Ophthalmology \& Optometry and Eye Hospital, Wenzhou Medical University, China, for his kind help with the statistics support. The authors would like to thank Peng Shi from Medical Statistics Department, Children's Hospital of Fudan University, Shanghai, China, for his kind help with the sample size correction and the statistics support. The authors would also like to thank all research assistants, optometrists, technicians and nursing staff involved, who contributed to the practical organization and execution of this study.

\section{Compliance with ethical standards}

Conflict of interest Victor Chong is a consultant for Quantel Medical. The other authors declare that they have no conflict of interest.

Publisher's note Springer Nature remains neutral with regard to jurisdictional claims in published maps and institutional affiliations.

Open Access This article is licensed under a Creative Commons Attribution 4.0 International License, which permits use, sharing, adaptation, distribution and reproduction in any medium or format, as long as you give appropriate credit to the original author(s) and the source, provide a link to the Creative Commons license, and indicate if changes were made. The images or other third party material in this article are included in the article's Creative Commons license, unless indicated otherwise in a credit line to the material. If material is not included in the article's Creative Commons license and your intended use is not permitted by statutory regulation or exceeds the permitted use, you will need to obtain permission directly from the copyright holder. To view a copy of this license, visit http://creativecommons. org/licenses/by/4.0/.

\section{References}

1. Liew G, Quin G, Gillies M, Fraser-Bell S. Central serous chorioretinopathy: a review of epidemiology and pathophysiology. Clin Exp Ophthalmol. 2013;41:201-14.

2. Gemenetzi M, De Salvo G, Lotery AJ. Central serous chorioretinopathy: an update on pathogenesis and treatment. Eye. 2010;24:1743-56.

3. Yannuzzi LA, Slakter JS, Gross NE, Spaide RF, Costa D, Huang $\mathrm{SJ}$, et al. Indocyanine green angiography-guided photodynamic therapy for treatment of chronic central serous chorioretinopathy: a pilot study. Retina. 2003;23:288-98.

4. Lim JI, Glassman AR, Aiello LP, Chakravarthy U, Flaxel CJ, Spaide RF, et al. Collaborative retrospective macula society study of photodynamic therapy for chronic central serous chorioretinopathy. Ophthalmology. 2014;121:1073-8.

5. Palanker D, Blumenkranz MS. Retinal laser therapy: biophysical basisand applications. In: Ryan S, Wilkinson H, Sadda W, editors. Ch. 39. RETINA. 5th ed. Vol. 1. Elsevier Inc; 2013, p. 752-5.

6. Khosla PK, Rana SS, Tewari HK, Azad RU, Talwar D. Evaluation of visual function following argon laser photocoagulation in central serous retinopathy. Ophthalmic Surg Lasers. 1997;28:693-7.

7. Schatz H, Yannuzzi LA, Gitter KA. Subretinal neovascularization following argon laser photocoagulation treatment for central serous chorioretinopathy: complication or misdiagnosis? Trans Sect Ophthalmol Am Acad Ophthalmol Otolaryngol. 1977;83:893-906.

8. Maruko I, Koizumi H, Hasegawa T, Arakawa H, Iida T. Subthreshold $577 \mathrm{~nm}$ micropulse laser treatment for central serous chorioretinopathy. PLoS ONE. 2017;12:e0184112.

9. Kim YK, Ryoo NK, Woo SJ, Park KH. Comparison of visual and anatomical outcomes of half-fluence and half-dose photodynamic 
therapy in eyes with chronic central serous chorioretinopathy. Graefes Arch Clin Exp Ophthalmol. 2015;253:2063-73.

10. Cardillo Piccolino F, Eandi CM, Ventre L, Rigault de la Longrais RC, Grignolo FM. Photodynamic therapy for chronic central serous chorioretinopathy. Retina. 2003;23:752-63.

11. Chan WM, Lai TY, Lai RY, Tang EW, Liu DT, Lam DS. Safety enhanced photodynamic therapy for chronic central serous chorioretinopathy: one-year results of a prospective study. Retina. 2008;28:85-93.

12. Chan WM, Lai TY, Lai RY, Liu DT, Lam DS. Half-dose verteporfin photodynamic therapy for acute central serous chorioretinopathy: one-year results of a randomized controlled trial. Ophthalmology. 2008;115:1756-65.

13. Brader HS, Young LH. Subthreshold diode micropulse laser: a review. Semin Ophthalmol. 2016;31:30-39.

14. Breukink MB, Downes SM, Querques G, van Dijk EHC, den Hollander AI, Blanco-Garavito R, et al. Comparing half-dose photodynamic therapy with high-density subthreshold micropulse laser treatment in patients with chronic central serous chorioretinopathy (the PLACE trial): study protocol for a randomized controlled trial. Trials. 2015;16:419.

15. Roisman L, Magalhaes FP, Lavinsky D, Moraes N, Hirai FE, Cardillo JA, et al. Micropulse diode laser treatment for chronic central serous chorioretinopathy: a randomized pilot trial. Ophthalmic Surg Lasers Imaging Retina. 2013;44:465-70.

16. Koss MJ, Beger I, Koch FH. Subthreshold diode laser micropulse photocoagulation versus intravitreal injections of bevacizumab in the treatment of central serous chorioretinopathy. Eye. 2012;26:307-14.

17. Kretz FT, Beger I, Koch F, Nowomiejska K, Auffarth GU, Koss MJ. Randomized clinical trial to compare micropulse photocoagulation versus half-dose verteporfin photodynamic therapy in the treatment of central serous chorioretinopathy. Ophthalmic Surg Lasers Imaging Retin. 2015;46:837-43.

18. Chen SN, Hwang JF, Tseng LF, Lin CJ. Subthreshold diode micropulse photocoagulation for the treatment of chronic central serous chorioretinopathy with juxtafoveal leakage. Ophthalmology. 2008;115:2229-34.

19. Gupta B, Elagouz M, McHugh D, Chong V, Sivaprasad S. Micropulse diode laser photocoagulation for central serous chorioretinopathy. Clin Exp Ophthalmol. 2009;37:801-5.

20. Lanzetta P, Furlan F, Morgante L, Veritti D, Bandello F. Nonvisible subthreshold micropulse diode laser $(810 \mathrm{~nm})$ treatment of central serous chorioretinopathy. A pilot study. Eur J Ophthalmol. 2008;18:934-40.
21. Scholz P, Ersoy L, Boon CJ, Fauser S. Subthreshold micropulse laser $(577 \mathrm{~nm})$ treatment in chronic central serous chorioretinopathy. Ophthalmologica. 2015;234:189-94.

22. Kim JY, Park HS, Kim SY. Short-term efficacy of subthreshold micropulse yellow laser (577-nm) photocoagulation for chronic central serous chorioretinopathy. Graefes Arch Clin Exp Ophthalmol. 2015;253:2129-35.

23. Yadav NK, Jayadev C, Mohan A, Vijayan P, Battu R, Dabir S, et al. Subthreshold micropulse yellow laser $(577 \mathrm{~nm})$ in chronic central serous chorioretinopathy: safety profile and treatment outcome. Eye. 2015;29:258-64. quiz 265

24. Scholz P, Altay L, Fauser S. Comparison of subthreshold micropulse laser $(577 \mathrm{~nm})$ treatment and half-dose photodynamic therapy in patients with chronic central serous chorioretinopathy. Eye. 2016;30:1371-7.

25. Joondeph BC, Joondeph HC, Blair NP. Retinal macroaneurysms treated with the yellow dye laser. Retina. 1989;9:187-92.

26. Mainster MA. Decreasing retinal photocoagulation damage: principles and techniques. Semin Ophthalmol. 1999;14:200-9.

27. Korobelnik JF, Holz FG, Roider J, Ogura Y, Simader C, SchmidtErfurth U, et al. Intravitreal aflibercept injection for macular edema resulting from central retinal vein occlusion: one-year results of the phase 3 GALILEO study. Ophthalmology. 2014;121:202-8.

28. Early Treatment Diabetic Retinopathy Study Research Group. Grading diabetic retinopathy from stereoscopic color fundus photographs-an extension of the modified Airlie House classification. ETDRS report number 10. Ophthalmology. 1991;98(5 Suppl):786-806.

29. Spaide RF, Koizumi H, Pozzoni MC. Enhanced depth imaging spectral-domain optical coherence tomography. Am J Ophthalmol. 2008;146:496-500.

30. Ricci F, Missiroli F, Regine F, Grossi M, Dorin G. Indocyanine green enhanced subthreshold diode-laser micropulse photocoagulation treatment of chronic central serous chorioretinopathy. Graefes Arch Clin Exp Ophthalmol. 2009;247:597-607.

31. Malik KJ, Sampat KM, Mansouri A, Steiner JN, Glaser BM. Low-intensity/high-density subthreshold microPulse diode laser for chronic central serous chorioretinopathy. Retina. 2015;35:532-6.

32. Arsan A, Kanar HS, Sonmez A. Visual outcomes and anatomic changes after sub-threshold micropulse yellow laser (577-nm) treatment for chronic central serous chorioretinopathy: long-term follow-up. Eye. 2018;32:726-33. 